\title{
Equitable partnerships in global health research
}

To the Editor - Equitable partnerships are essential for global health research. However, the field is dominated by researchers from the global north and this imbalance results from entrenched power asymmetries (often linked to funding sources) that can undermine the contributions and knowledge of local experts. Some funders promote equity in the way resources are distributed across partner organizations - weighting the spend towards the global south and appointing principal investigators from the global south. These efforts are not enough, however, and academics from low- and middle-income countries are still underrepresented in the global health literature ${ }^{1}$. Therefore, it is important for researchers to take the initiative to ensure that equitable, mutually supportive partnerships are developed from the generation of the initial research concept through to the project delivery and final dissemination of the research outcomes.

Based on a growing body of literature on the principles of developing equitable partnerships $^{2-4}$, we present a framework comprised of four pillars: co-creation, communication, commitment and continuous review (Fig. 1), which also includes the values of the Global Code of Conduct for Research in Resource-Poor Settings $^{2}$ - fairness, respect, care and honesty. We have formally adopted these principles in our own collaboration between the UK and Pakistan over the last fifteen years on micronutrient deficiencies ${ }^{5,6}$ and we would encourage colleagues to establish a similar framework to foster such a mindset when embarking upon collaborations wherever there is the potential for inequity, whether this be in international, or within sub-national contexts.

\section{Co-creation}

Equity is not just about creating a level playing field for partners to work together, but also means understanding and compensating for inherent inequalities to enable all partners to fully participate and benefit from interactions. This applies to the co-creation of the research questions to ensure local challenges are addressed. Involving beneficiaries of research as both participants and partners in the research project encourages equitability and engagement. Fairness is key to the distribution of financial resources, and the contribution to and credit for research outputs. Many funding organizations look for evidence of co-creation of the research question by all partners. It is critical that all funders look for evidence of co-creation, and where possible, facilitate opportunities for potential partners to refine the research questions together, as part of the funding process.

Co-creation of research question. Research questions should be developed in response to the local needs as expressed by the community. In one of our first collaborative projects exploring the barriers that mothers face in providing nutritious meals for their infants and children, interviews and focus group discussions with health visitors and mothers attending antenatal services at a rural emergency satellite hospital inspired the idea of setting up a demonstration kitchen at the hospital - a space where mothers could come to receive basic education around safe food preparation, weaning practices and the benefits of diversifying the diet, share and prepare food together, and socialize together, while facilitating research ${ }^{7}$. We seek to ensure that infrastructure resources are used in a way that both serves the community and serves the research. The combination of quantitative and qualitative approaches places equity at the heart of the relationship between researcher and research participant, ensuring that all voices contribute to the identification and solution of the research question.

Co-creation of study implementation. As partners and stakeholders in the research project, community members can also be instrumental in the development and operationalization of the data collection protocol. Some examples of this we have found in the area of community nutrition interventions include identification of eligible households for participation in the study, recruitment of local women to join the field team to assist with data collection, logistics around appropriate gender segregation and access to schools to interview the participating adolescent girls. This concept of community involvement is well established in medical research ${ }^{8}$.

Co-creation of research outputs. For fairness in representation in the literature, norms and expectations around academic authorship need to be clarified early in the partnership. There are a number of different rubrics that are used for deciding on the inclusion criteria for authors and the order in which the authors are listed. We recommend the guidance on authorship

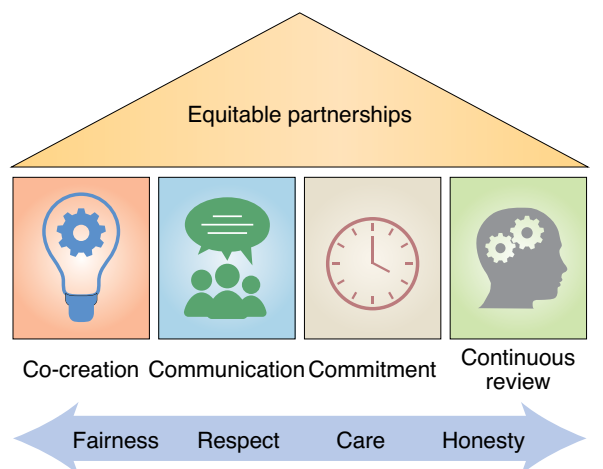

Fig. 1 | Framework for the development of equitable partnerships. Equitable partnerships are supported by the four pillars of co-creation, communication, commitment and continuous review. Threading through all the pillars are the values of fairness, respect, care and honesty, as described in the Global Code of Conduct for Research in Resource-Poor Settings ${ }^{2}$.

provided by the International Committee of Medical Journal Editors ${ }^{9}$ with all of the options for the order as stated by Tscharntke et al. ${ }^{10}$ to devise a transparent and adjustable plan, including an agreement to explicitly state which approach has been adopted within the acknowledgements section of each publication.

Dissemination of research findings to communities and stakeholders is a keystone of equitable partnerships. Laying the ground in advance with a communication plan feeds into the virtuous cycle of trust between project partners.

\section{Communication}

Equitable partnerships are built upon mutual understanding and respect for cultural norms, including religious, cultural and societal boundaries. One way to develop a greater cultural awareness in the study location is to create a map of local power structures and communication pathways within and beyond the local communities. Our work, for example, is conducted near Peshawar, in what was formerly part of a tribal society with traditional and conservative values. Decisions are made on behalf of the community by Jirga - groups of male elders from each village who are trusted and respected by the community and whose decisions filter down to household level. Likewise, problems or concerns at the household level are escalated, discussed and resolved by the Jirga. Involving the Jirga at 
regular intervals during the development of our work ensured our methods were feasible and culturally acceptable ${ }^{11}$.

\section{Commitment}

There is often very little time between the announcement of research funding calls and their deadlines, and there is a temptation to seek partners rapidly. Some funding organizations provide partner-finding websites to facilitate the rapid identification of relevant and willing research partners in a given field. We favour an incremental approach, where developing equitable partnerships requires patience, building trust and long-term commitment. Beginning with the co-creation of the research needs between partners, small amounts of local funding may enable some formative work to be undertaken such that when larger funding opportunities arise an established track record can be evidenced. Partnerships then develop in line with the complexity of the research projects undertaken, and new partners can join the consortium to broaden the expertise base and enable interdisciplinary and transdisciplinary research. Introducing new partners requires careful management to ensure that the central ethos of a community-led approach is maintained as the projects become more complex and the budgets greater.

Long-term commitment to the partnership involves building and investing in research capacity for the future including training. The training received by community field workers, postgraduate and postdoctoral research assistants and the opportunity to learn from national and international experts has enabled all staff to broaden their skill base and improve future opportunities.

\section{Continuous review}

A continuous process of review and consultation is necessary to develop and refine the equitable partnerships research model. Successful long-term partnerships are not static, they evolve and flex in response to changes in the funding landscape and research priorities. Furthermore, shocks such as the COVID-19 pandemic present additional challenges: emphasized social inequalities between project partner countries, ethical considerations of how and when to re-start laboratory and field work from different partner perspectives, and consensus on the way forward for the wellbeing of researchers and communities must all be navigated. Honesty in reflection and the evaluation of successes and failures is part of this process. Many projects have monitoring and evaluation formally built in to the study protocol a priori - but many do not. Irrespective of this, it is good practice to regularly consult with all partners regarding the research process - not just at the end of the project, but also while the research is underway so that adjustments can be made, and hazards averted. Like any relationship, an equitable partnership requires continuous attention to flourish and grow.

Malnutrition, in whatever form, affects every nation of the globe, and our food systems are interdependent. In this, the decade of action on nutrition, greater cooperation between researchers and institutions in the global north and global south on food systems is paramount. It is crucial that an incremental approach to building research consortia, with pillars of co-creation, communication, commitment and continuous review, sets equity and an ethos of fairness in stone for research, and for researchers.

There is an important role for funders too, in stipulating that equitable partnerships are embedded in programmes they fund. They too must review their own processes and procedures to ensure that their own organizations model this way of working.

Mukhtiar Zaman', Gulman Afridi², Heather Ohly ${ }^{3}$, Harry J. McArdle ${ }^{4}$ and
Nicola M. Lowe (iD) 3 瓜

${ }^{1}$ Department of Pulmonology, Rehman Medical Institute, Peshawar, Pakistan. ${ }^{2}$ Abaseen Foundation, Peshawar, Pakistan. ${ }^{3}$ UCLan Research Centre for Global Development, University of Central Lancashire, Preston, UK. ${ }^{4}$ School of Biosciences, University of Nottingham, Sutton Bonington Campus, Sutton Bonington, UK.

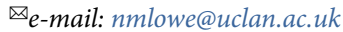

Published online: 7 December 2020

https://doi.org/10.1038/s43016-020-00201-9

References

1. Lyer, A. R. Lancet Glob. Health 6, E142 (2018).

2. Global Code of Conduct for Research in Resource-Poor Settings (TRUST Consortium, 2018).

3. Finding and Building Effective and Equitable Research Collaborations or Partnerships (UKCDR, 2017).

4. Promoting Fair and Equitable Research Partnerships to Respond to Global Challenges (UKRI, 2018).

5. Ohly, H. et al. Nutr. Bull. 44, 60-64 (2019).

6. Lowe, N. M. et al. BMJ Open 8, e021364 (2018).

7. Lhussier, M., Dykes, F., Bagash, S. A., Zaman, M. \& Lowe, N. Health Promot. Int. 27, 454-462 (2011).

8. Fregonese, F. BMC Med. Ethics 19, 44 (2018).

Defining the Role of Authors and Contributors (ICMJE, 2020).

10. Tscharntke, T., Hochberg, M. E., Rand, T. A., Resh, V. H. \& Krauss, J. PLoS Biol. https://doi.org/10.1371/journal.pbio. 0050018 (2007).

11. Mahboob, U. et al. Pilot Feasibility Stud. 6, 117 (2020).

Acknowledgements

The authors would like to acknowledge the valuable input of all our project partners and stakeholders, in particular our study communities who have willingly worked with us over the last 20 years. We would particularly like to acknowledge the tireless work of the members of the Board of Governors at AF PK, particularly O. Ullah, and the Trustees of the AF UK, particularly H. Bingley OBE, who have been instrumental in the success of the consortium.

\section{Author contributions}

We have followed the 'first-last' author emphasis approach for the order of the authors listed ${ }^{10}$. This approach highlights the importance of the first and last authors. M.Z. and N.M.L. have led the development of research partnerships since 2003. M.Z. and G.A. play a central role in stakeholder engagement and community liaison. H.J.M. and H.O. are members of the BIZIFED research consortium. All authors contributed to the drafting of this Correspondence.

Competing interests

The authors declare no competing interests. 